\title{
Sonographically Guided Knee Meniscus Injections: Feasibility, Techniques, and Validation
}

\author{
Michael R. Baria, MD/MBA, Jacob L. Sellon, MD, Dan Lueders, MD, Jay Smith, MD
}

\begin{abstract}
Background: There is a growing interest in the use of biologic agents such as platelet-rich plasma and mesenchymal stem/stromal cells to treat musculoskeletal injuries, including meniscal tears. Although previous research has documented the role of diagnostic ultrasound to evaluate meniscal tears, sonographically guided (SG) techniques to specifically deliver therapeutic agents into the meniscus have not been described.

Objective: To describe and validate SG injection techniques for the body and posterior horn of the medial and lateral meniscus. Design: Prospective, cadaveric laboratory investigation.

Setting: Academic institution procedural skills laboratory.

Subjects: Five unenbalmed cadaveric knee-ankle-foot specimens from 5 donors ( 3 female and 2 male) ages $33-92$ years (mean age 74 years) with body mass indices of $21.1-32.4 \mathrm{~kg} / \mathrm{m}^{2}$ (mean $24.1 \mathrm{~kg} / \mathrm{m}^{2}$ ).

Methods: A single, experienced operator completed SG injections into the bodies and posterior horns of the medial and lateral menisci of 5 unenbalmed cadaveric knees using colored latex and a 22-gauge, 38-mm needle. After injection, coinvestigators dissected each specimen to assess latex distribution within the menisci and identify injury to intra-articular and periarticular structures.

Main Outcome Measures: Latex location within the target region of meniscus (accurate/inaccurate), and iatrogenic injury to "at risk" intra- and periarticular structures (present/absent).

Results: Seventeen of 20 injections were accurate. Two of 3 inaccurate injections infiltrated the posterior horn of the medial meniscus instead of the targeted meniscal body. One inaccurate lateral meniscus injection did not contain latex despite sonographically accurate needle placement. No specimen exhibited injury to regional neurovascular structures or intra-articular hyaline cartilage.

Conclusions: SG meniscus injections are feasible and can accurately and safely deliver injectates such as regenerative agents into bodies and posterior horns of the medial and lateral menisci. The role of SG intrameniscal injections in the treatment of patients with degenerative and traumatic meniscal disorders warrants further exploration.

Level of Evidence: Not applicable.
\end{abstract}

\section{Introduction}

Knee meniscal injuries are very common, may significantly impact function, and predispose the knee to accelerated osteoarthritis [1]. The body and posterior horn of the meniscus are particularly susceptible to acute injury and chronic degeneration [2]. Symptomatic meniscal tears may be treated with activity modification, modalities, bracing, therapeutic exercise, intraarticular injections and, when clinically indicated, surgical debridement or repair [3].

In recent years, there has been increased interest in the use of biologic agents (eg, platelet-rich plasma, bone marrow aspirate concentrate, and mesenchymal stem/stromal cells [MSCs]) to treat patients suffering from a variety of musculoskeletal disorders, including tendinosis, osteoarthritis, and meniscal tears [4,5]. Vangsness et al [6] found that a single, postoperative intra-articular injection of culture expanded, allogeneic, bone marrow-derived MSCs was well tolerated, improved pain, and promoted meniscal regeneration in patients after arthroscopic debridement of degenerative meniscal tears. Multiple authors also have reported therapeutic benefits from direct placement of biologic agents (eg, fibrin clots, stem cell-scaffold combinations) into the meniscus as an adjunct to arthroscopic surgery 
$[7,8]$. Although intra-articular injections of culture expanded, autologous, bone marrow-derived MSCs also may improve symptoms and possibly promote meniscal regeneration in the context of nonoperative treatment programs for degenerative meniscal tears [9], the feasibility of percutaneous intrameniscal delivery of biologic agents has not been explored previously.

The ability of high-resolution ultrasound (US) to image the meniscus has been well-established [10-12]; however, the feasibility of using sonographic guidance (SG) to specifically deliver needles/injectates into the bodies and posterior horns of the medial and lateral menisci has not been evaluated formally. Consequently, the primary purpose of this investigation was to determine the feasibility of SG intrameniscal injections; more specifically, to determine the safety and accuracy of SG body and posterior horn intrameniscal injections by using an unembalmed cadaveric model. We hypothesized that SG could be used to accurately and safely place a standard 22-gauge needle into the bodies and posterior horns of the medial and lateral menisci. Clinically, the results of this investigation would provide a foundation for the clinical implementation of SG intrameniscal injections in appropriately selected patients.

\section{Methods}

\section{General Design}

A single, experienced operator (the corresponding author) injected $0.5-1.0 \mathrm{~mL}$ of colored latex dye (Ward's Biological Science, Rochester, NY) into the bodies and posterior horns of both the medial and lateral menisci in 5 unembalmed, cadaveric knee-ankle-foot specimens using direct SG and a 22 -gauge, $38-\mathrm{mm}$ needle. At the time of the investigation, the operator had more than 12 years of experience in diagnostic and interventional musculoskeletal US, including SG knee injections. All specimens were obtained from the Department of Anatomy's Foundation Bequest Program and were free from deformity, signs of trauma, and postsurgical change. Study coinvestigators dissected each specimen to determine the accuracy of intrameniscal latex injection as well as the presence or absence of iatrogenic injury to the regional neurovascular structures and intra-articular hyaline cartilage. This investigation was approved by the Bio-Specimens Subcommittee of the Institutional Review Board at the authors' institution (Institutional Review Board no. 14-005967).

\section{Equipment}

All imaging and injections were completed with a Philips iU22 ultrasound machine and a 12-5 MHz linear array transducer (Philips Ultrasound Systems, Bothell, WA). All injections were completed with standard 22-gauge, 38-mm stainless-steel needles.

\section{Injection Procedure}

Each specimen was placed in a side-lying position with the target side of the knee facing the ceiling. To optimize sonographic visualization and meniscal access, the knee was flexed to $30-60^{\circ}$ and a gravity-induced valgus or varus stress was imparted on the joint by placing a small, rolled-up towel under the dependent side of the knee [11]. The transducer was then placed in an anatomical coronal plane across the joint, perpendicular to the long axis (LAX) of the meniscus, yielding a short-axis view (or cross-section) of the meniscus. Thereafter, the regional "at-risk" structures were identified sonographically and their locations were marked with an indelible ink marker-the saphenous nerve medially and the common fibular nerve laterally.

The medial and lateral meniscal body injections were conducted via a similar technique. After identification of the regional "at-risk" structures, the meniscal body was reidentified in the coronal plane (short-axis meniscal view). For the medial meniscal body, a 22gauge, $38-\mathrm{mm}$ stainless-steel needle was then advanced with a SG, out-of-plane, anterior-to-posterior approach. During the first 3 injections, the needle entry point and trajectory were chosen primarily based on avoiding the saphenous nerve. However, after the documentation of 2 inaccurate injections placed into the posterior horn (as described in the Results section), the medial meniscal body injection technique was modified to use the medial collateral ligament (MCL) as a consistent landmark. For these subsequent injections, the transducer was placed in the coronal plane just anterior to the MCL to facilitate consistent needle and latex placement into the body of the medial meniscus using the out-of-plane approach. Once the needle tip was visualized within the meniscal body, the transducer was rotated $90^{\circ}$ into the anatomical axial plane, yielding an LAX view of the meniscus and an in-plane view of the needle (Figure 1). The needle was advanced to the innermost/deepest portion of the meniscal body using direct SG and an in-plane approach. After final needle placement, the operator injected $0.5-1.0 \mathrm{~mL}$ of colored latex dye into the meniscus as the needle was withdrawn. The lateral meniscal body was injected via a similar technique, with the transducer placed in an anatomical coronal plane at the level of popliteus sulcus.

To inject the posterior horns of the menisci, the meniscal body initially was identified as described previously. Thereafter, the transducer was translated posteriorly over the region of the posterior horn. However, in contrast to the meniscal body injection technique, before needle entry the transducer was rotated to provide a LAX view of the posterior horn. Pilot investigations had indicated that visualizing the posterior horn in its LAX throughout the procedure facilitated identification and maintenance of the necessary 

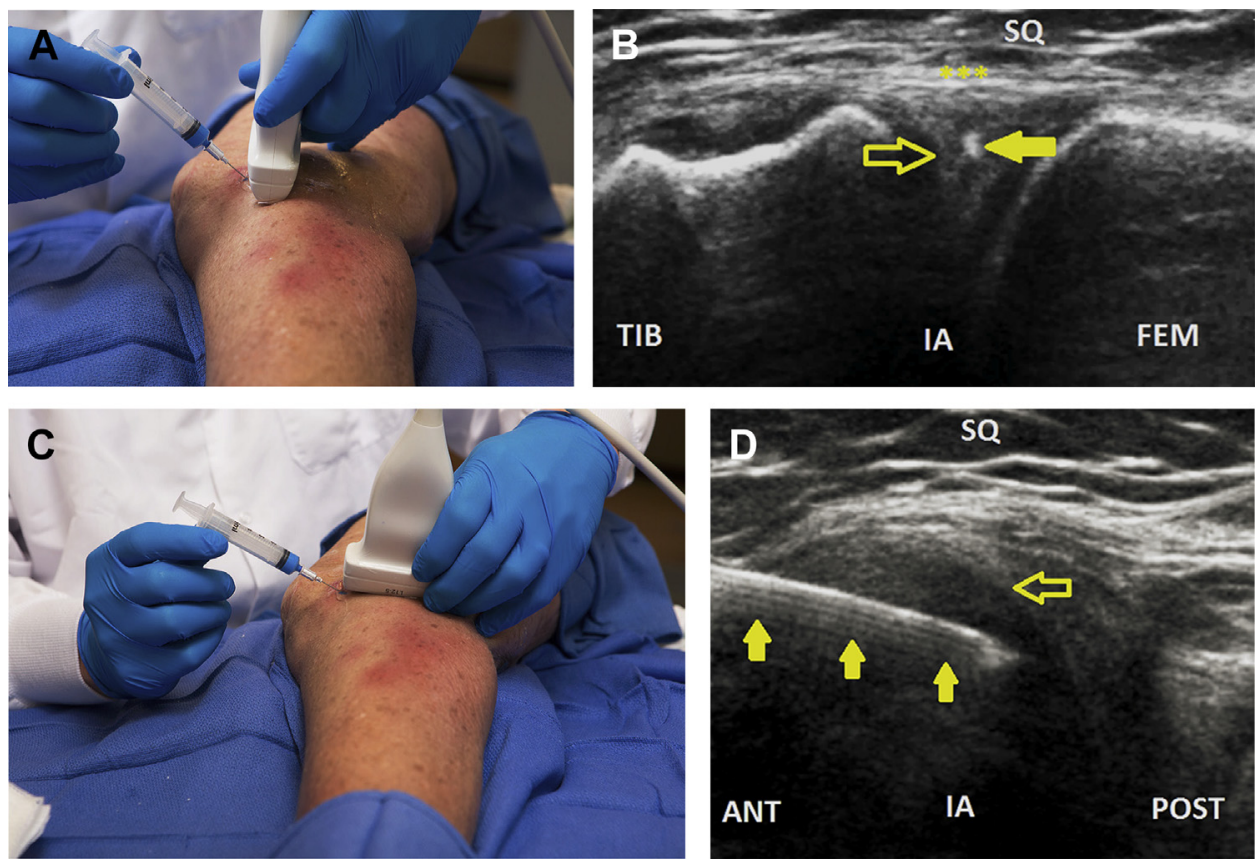

Figure 1. (A) Setup for a SG out-of-plane right medial meniscus body injection showing probe and needle position in an unembalmed cadaveric specimen. (B) Short-axis view of the medial meniscus body (hollow yellow arrow) demonstrating a medial meniscus body injection with a 22 -gauge stainless-steel needle. The needle (solid yellow arrow) is initially positioned using an out-of-plane approach, entering anterior to the MCL (asterisks) and advanced posteriorly. (C) Setup for medial meniscus body injection after probe rotation into a long-axis view of the medial meniscus and in-plane view of the needle. (D) Long-axis view of the medial meniscus body (hollow yellow arrow) and an in-plane view of the needle (solid yellow arrows). ANT = anterior; FEM = femur; IA = intra-articular space; $M C L=$ medial collateral ligament; $P O S T=$ posterior; $S Q=$ subcutaneous tissue; TIB = tibia.

trajectory to place the needle into the deeper portions of the posterior horn. After sonographic visualization of the posterior horn, a 22-gauge, 38-mm stainless-steel needle was advanced using an in-plane, anterior-toposterior approach. The needle tip was advanced to the deepest visible aspect of the posterior horn (Figure 2) and $0.5-1.0 \mathrm{~mL}$ of latex dye was injected as the needle was withdrawn.

After each injection, coinvestigators dissected the specimen to determine injection accuracy and assess for iatrogenic injury to the regional "at-risk" structures. Accuracy was scored as either "accurate" (a clear track of latex dye within the targeted meniscus region) or "inaccurate" (no clear track seen within the targeted meniscus region). Qualitative observations included the quality of latex spread within the target region, the presence of latex overflow into adjacent regions, and the presence of iatrogenic injury to the regional "atrisk" structures including the saphenous nerve, common fibular nerve, lateral inferior geniculate artery, and
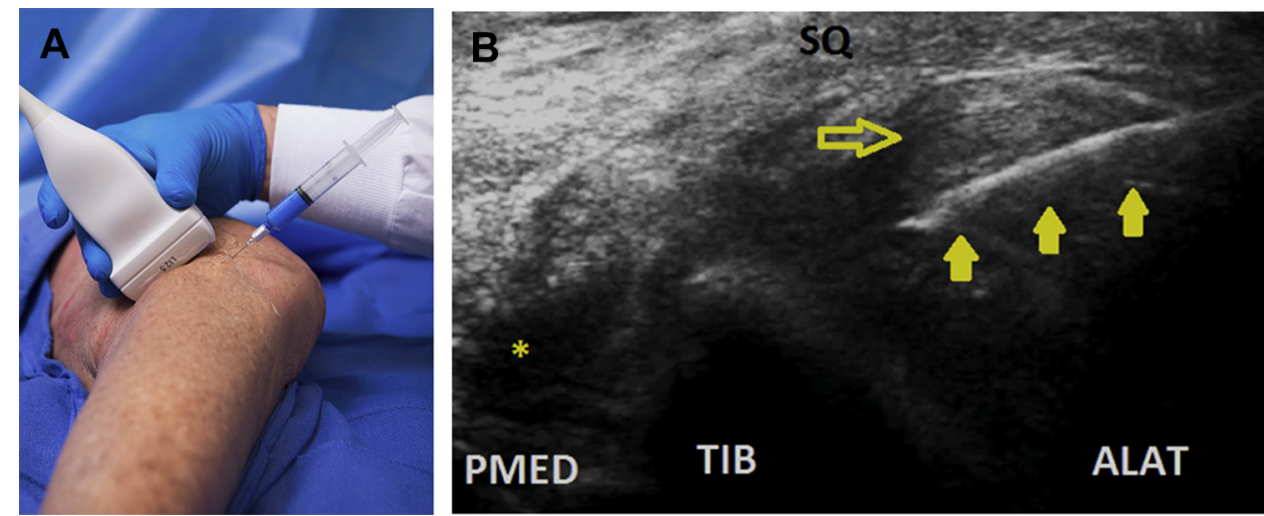

Figure 2. (A) Setup for a SG in-plane right lateral meniscus posterior horn injection, showing probe and needle position in an unembalmed cadaveric specimen. (B) LAX view of the lateral meniscus posterior horn (hollow yellow arrow) demonstrating injection with a 22 -gauge stainless steel needle (solid yellow arrows). Needle is advanced in an anterolateral to posteromedial direction. Asterisk indicates the posterior cruciate ligament (anisotropic). ALAT = anterolateral; PMED = posteromedial; SQ = subcutaneous tissue; $\mathrm{TIB}=$ tibia. 
intra-articular hyaline cartilage. All outcomes were graded by a consensus of 2 coinvestigators. Results were summarized with descriptive statistics.

\section{Results}

A total of 20 SG intrameniscal injections were performed in 5 unembalmed cadaveric knees from 5 donors ( 3 male, 2 female) ages 33-92 years (mean 74 years) and body mass indices of $21.1-32.4 \mathrm{~kg} / \mathrm{m}^{2}$ (mean $24.1 \mathrm{~kg} /$ $\mathrm{m}^{2}$ ). Sonographic visualization of the meniscal body and posterior horn, saphenous nerve, and common fibular nerve was satisfactory in all specimens. Not unexpectedly, sonographic visualization of the lateral inferior geniculate artery was not possible in all specimens because of the absence of pulsatile blood flow. Needle visualization was excellent in all specimens and 2 general injection flow patterns were observed: (1) high resistance flow, in which latex distribution was more discrete and limited to the needle track, and (2) low resistance flow, in which the latex distribution was more irregular and extended into the meniscus beyond the needle track. Although formal sonographic assessment of meniscal status was not performed as part of this investigation, the former flow pattern was more common in normal menisci, whereas the latter was observed in association with degenerative changes and/or meniscal clefts potentially representing tears.

Injection accuracy is presented in Table 1 . Seventeen of 20 injections were accurate. Two of the 3 inaccurate injections were medial meniscal body injections in which the latex was placed more posteriorly than desired -in the region of the posterior horn (Figure 3 ). This was determined to result from initial placement of the transducer just superficial to the MCL. With this transducer placement, during the out-of-plane needle approach the needle had a tendency to pass too far posteriorly (ie, out of the region of the body and into the region of the posterior horn) by the time it reached its target depth. This technical limitation was resolved by placing the transducer anterior to the $M C L$ during subsequent meniscal body injections. The third

Table 1

Injection accuracy

\begin{tabular}{|c|c|c|c|}
\hline Meniscus Region & $\begin{array}{l}\text { Accurate } \\
\text { (n) }\end{array}$ & $\begin{array}{l}\text { Inaccurate } \\
\text { (n) }\end{array}$ & Observation \\
\hline $\begin{array}{l}\text { Medial meniscus } \\
\text { body }\end{array}$ & 3 & 2 & $\begin{array}{l}\text { Latex posterior to } \\
\text { target }- \text { in } \\
\text { posterior horn }\end{array}$ \\
\hline $\begin{array}{l}\text { Medial meniscus } \\
\text { posterior horn }\end{array}$ & 5 & 0 & \\
\hline $\begin{array}{l}\text { Lateral meniscus } \\
\text { body }\end{array}$ & 4 & 1 & No latex deposition \\
\hline $\begin{array}{l}\text { Lateral meniscus } \\
\text { posterior horn }\end{array}$ & 5 & 0 & \\
\hline Total & 17 (85\%) & $3(15 \%)$ & \\
\hline
\end{tabular}

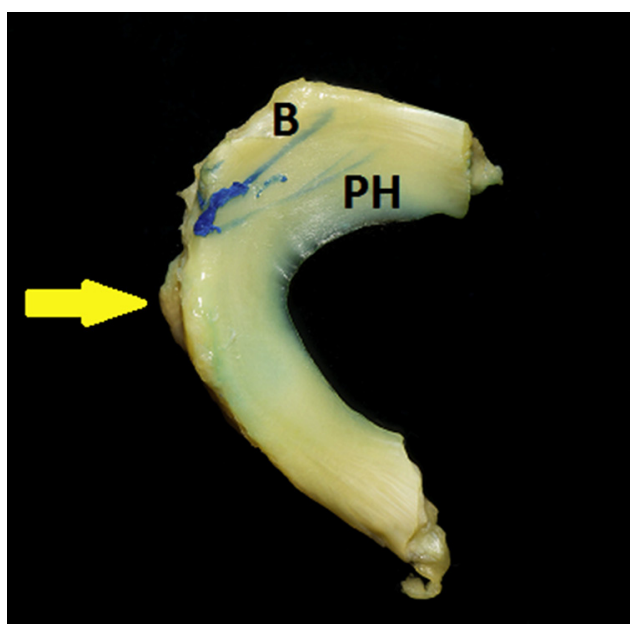

Figure 3. Superior view of the medial meniscus demonstrating an accurately placed latex trail in the posterior horn $(\mathrm{PH})$; however, the meniscal body injection (B) was placed too far posteriorly and therefore was considered inaccurate. The injection technique was subsequently modified to prevent this "overshoot" by placing the transducer just anterior to the MCL (solid yellow arrow, see text for explanation). Top $=$ posterior; Bottom $=$ anterior; Left $=$ medial $/$ superficial; Right $=$ lateral/deep.

inaccurate injection was a lateral meniscus body injection in which no latex was seen in the meniscus. No specimens demonstrated evidence of iatrogenic injury to the saphenous nerve, common fibular nerve, lateral inferior geniculate artery, or intra-articular hyaline cartilage.

\section{Discussion}

The most important finding of this investigation was that SG intrameniscal injections are feasible and can safely place injectates into the bodies and posterior horns of the medial and lateral menisci with acceptable accuracy. Consequently, the current results provide a foundation for consideration of SG intrameniscal injections in clinically appropriate populations.

Improving pain and function in patients with symptomatic degenerative or traumatic meniscal tears continues to challenge clinicians. Although surgical intervention is clearly indicated in selected cases, surgery is not without consequences. For example, Roos et al [13] demonstrated that when compared with agematched controls with healthy knees, patients treated with total meniscectomy had a relative risk of 14.0 for developing advanced radiographic findings of osteoarthritis and a 2-fold increased risk of developing clinically significant knee pain at a mean of 21 years postsurgery. These and similar findings have fostered the philosophy of meniscal preservation when managing symptomatic meniscal disorders. For example, multiple studies have emphasized the clinical and biomechanical benefits of partial versus total meniscectomy $[14,15]$. 
Even partial meniscectomy, however, is not without consequence. A prospective, randomized trial by Hede et al [16] demonstrated that only $62 \%$ of patients treated with partial meniscectomy had an excellent outcome at a median 7.8 years' follow-up, which was not statistically significantly different from the $52 \%$ excellent outcome in the total meniscectomy group. Consequently, as the evidence in support of meniscal preservation has accumulated, a variety of meniscal repair techniques have evolved to avoid or minimize the extent of meniscal resection. Many of these techniques have been augmented successfully by intraoperative biologic treatments such as fibrin clot application, trephination, meniscocapsular abrasion, and more recently application of cell-based therapies and scaffolds $[7,8]$. The primary goal of these adjunctive biologic treatments is to facilitate healing and preserve or perhaps regenerate the meniscus.

Although the intraoperative application of biologic agents in the treatment of meniscal tears is well established, the use of biologic agents as an adjunct to nonoperative meniscus tear treatment has been limited to a few select case reports [9]. One potential explanation for this discrepancy is the inability to precisely deliver the desired biologic agent into the meniscus in the absence of direct intraoperative visualization. As demonstrated in the current study, high-resolution US can fill this void by facilitating precise and accurate needle placement into the bodies and posterior horns of the medial and lateral menisci, the most common regions associated with acute and chronic symptomatic meniscal tears [2]. Consequently, the role of SG intrameniscal injections can now be explored via future research and collective clinical experience.

There are several limitations and technical factors that warrant further discussion in the context of the current study. First, clinicians should exercise appropriate caution when extrapolating the results of this cadaveric investigation to clinical scenarios. Although the current study documents the feasibility of SG meniscal injections, we are unable to comment on the clinical efficacy of intrameniscal injections with respect to specific therapeutic agents or the role of intra- versus extrameniscal injections in specific clinical circumstances. With respect to safety, we did not directly visualize the lateral inferior geniculate artery due to lack of Doppler flow. Although this artery can be easily visualized on US and therefore avoided when performing SG lateral meniscal injections, the current study design precludes definitive statements pertaining to the safety of SG injections with respect to the perimeniscal vasculature.

Second, we used a 22-gauge, 38-mm stainless-steel needle to accommodate the viscosity of the latex. We recognize that some therapeutic agents may have higher or lower viscosities that will influence the choice of needle gauge and the propensity for injectate spread from the target site postinjection. A low-viscosity injectable can be delivered via a small-gauge needle, thus minimizing inadvertent tissue trauma; however, the therapeutic agent may have a tendency to migrate from the target site. In comparison, a greater viscosity agent may more likely remain at the target site but would require a larger gauge needle and potentially incur additional risk of inadvertent tissue trauma. In addition to the needle gauge, we acknowledge the potential differences in viscosity between latex used in this study and that of various biologic agents used in clinical practice. That said, the purpose of this study was to validate injection technique. We used latex because of previous experience using this model in cadaveric studies. The advantages of latex for the study include ease of use and the ability to define a clear injection deposit on dissection. We recognize that the viscosity of latex may not be the same as biologic agents used in practice. There is no standard viscosity for various biologics because they may vary between patients and among different preparations. Therefore, it is impractical to test the flow characteristics of the "biologics" in the presented model. This limitation does not limit the applicability of the described injection technique. Operators will need to consider all these factors during any clinical application of SG meniscal injections.

Third, we reported 3 inaccurate injections, 2 of which resulted from technical factors that were mitigated by procedural changes as previously described (ie, placing the transducer anterior to rather than superficial to the MCL for the medial meniscus body injection). The reason for the third inaccurate injection remains indeterminate but may have resulted from the high latex viscosity. In the setting of normal menisci, which was the case with respect to the specimen in question, the latex injection required high injection pressure due to the densely packed meniscal fibers. Although the latex was "injected" as the needle was withdrawn, the flow of latex into the needle track could not be directly sonographically visualized in all cases. The use of small injection volumes and the compressibility of the latex solution further limited the ability to unequivocally confirm the actual delivery of intrameniscal latex during the injection. Consequently, we hypothesize that despite accurate needle placement as evidenced by the anatomically identified needle track, it is probable that no latex was in fact injected into the lateral meniscus in this case. We also recognize the less likely possibility that some latex was initially injected into the meniscus but subsequently flowed back out of the needle track as the result of high intrameniscal pressures. In either case, the lack of latex within the meniscus does not reflect a limitation of the SG intrameniscal injection since the needle was in fact placed accurately. Furthermore, from a clinical perspective, operators should not encounter these technical 
challenges when injecting menisci affected by acute or chronic tears, where low pressure distribution of the injectate is likely to be observed (Figure 4 versus Figures 5, 6, and 7).

Fourth, from our dissections it is clear that SG can be used to deliver injectates across the full width of the meniscus, including the innermost portion of the body (the white-white zone) and the root of the posterior horn (Figures 6 and 8, respectively). Therefore, SG intrameniscal injections could be considered to accurately deliver biologics to the avascular regions of the meniscus with poor spontaneous healing potential [17].

Fifth, the lateral meniscus was technically more difficult to inject than the medial meniscus because of its greater mobility. Specifically, as the needle was being placed into and advanced through the lateral meniscus, the meniscus often moved considerably. Meniscal degeneration or tearing may accentuate this phenomenon. Identifying mitigation strategies for this undesirable movement will require further investigation and experience.

Sixth, as exhibited in several of our specimens, injectate flow beyond the confines of the meniscus is possible and perhaps in some cases expected (Figures 4 and 7). Although the clinical significance of extrameniscal extension remains unknown and probably is dependent on the specific clinical scenario, extrameniscal extension can be minimized by precise needle placement and control facilitated by (1) practice in cadaveric specimens when available, (2) confirmation of needle tip position in 2 orthogonal planes, (3) avoidance of needle passage beyond the sonographically identified limits of the meniscus, (4) control of injection volume,

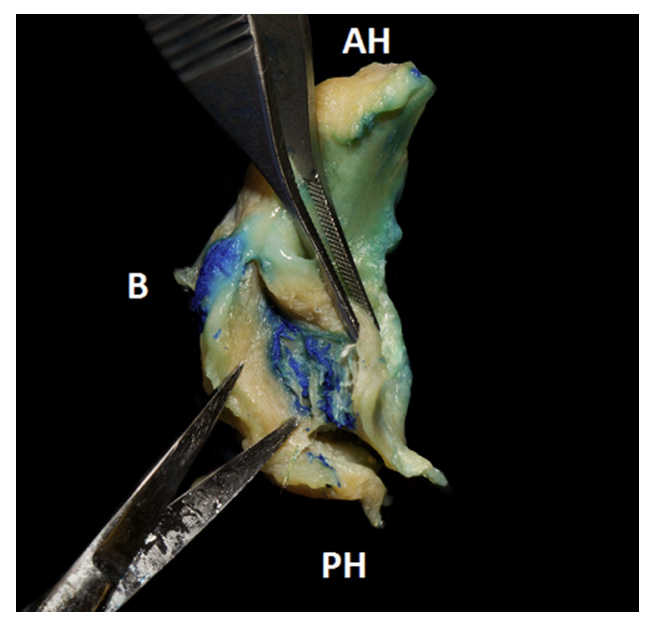

Figure 4. Superior view of medial meniscus posterior horn $(\mathrm{PH})$. The $\mathrm{PH}$ has been incised longitudinally and the superior half reflected to reveal the full injection pathway. Note the distribution of latex within the $\mathrm{PH}$. This meniscus exhibited structural changes consistent with degenerative meniscal tearing. Consequently, the latex spread throughout the region of degeneration. Left = superficial/medial; Right $=$ deep/lateral; Top $=$ anterior; Bottom $=$ posterior; $\mathrm{AH}=$ anterior horn; $\mathrm{B}=$ body.

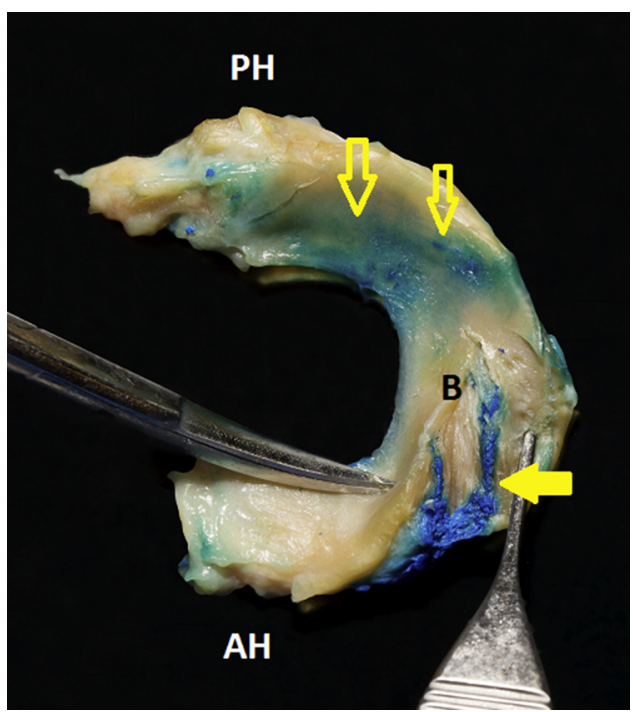

Figure 5. Superior view of the lateral meniscus body. Similar to Figure 4, the meniscus has been incised parallel to its long axis and the superior aspect of the meniscus body reflected to reveal the accurate meniscal body injection (solid yellow arrow). In comparison with Figure 4, this meniscus was grossly normal and the latex spread was therefore restricted to the needle track. Note also the staining from the accurately placed posterior horn $(\mathrm{PH})$ injection in this specimen (hollow yellow arrows). Left $=$ deep/medial; Right $=$ superficial/ lateral; Top $=$ posterior; Bottom $=$ anterior; $\mathrm{AH}=$ anterior horn; $\mathrm{B}=$ body.

and (5) the use of identifiable anatomical landmarks during the setup and execution of the procedure.

With respect to the latter recommendation, the anterior aspect of the MCL and the popliteal sulcus proved to be reliable landmarks for transducer placement and needle entry during medial and lateral body injections, respectively, whereas the posterior cruciate ligament $(\mathrm{PCL})$ also can be used to facilitate identification of the posterior horns of the menisci and if desired, the posterior roots [18]. The PCL initially is identified in an anatomical oblique sagittal plane and

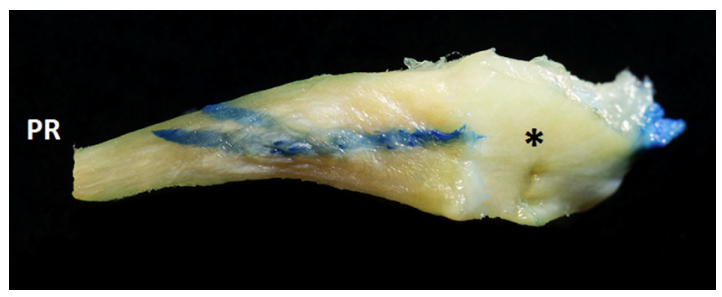

Figure 6. Cross-sectional view of the lateral meniscus posterior horn showing an accurate injection pathway. The specimen has been dissected to specifically demonstrate the posterior root (PR) of the lateral meniscus. The body and anterior horn have been resected to produce a meniscal cross section at the junction of body and posterior horn (asterisk), and a portion of the inner third (ie, white-white zone) of the posterior horn has been removed to demonstrate the injection track. Similar to the specimen in Figure 5, this meniscus was grossly normal, thus limiting latex flow to within the confines of the needle track (compare with Figure 4). Left $=$ deep/medial; Right $=$ superficial/lateral; Top $=$ cranial; Bottom $=$ caudal. 


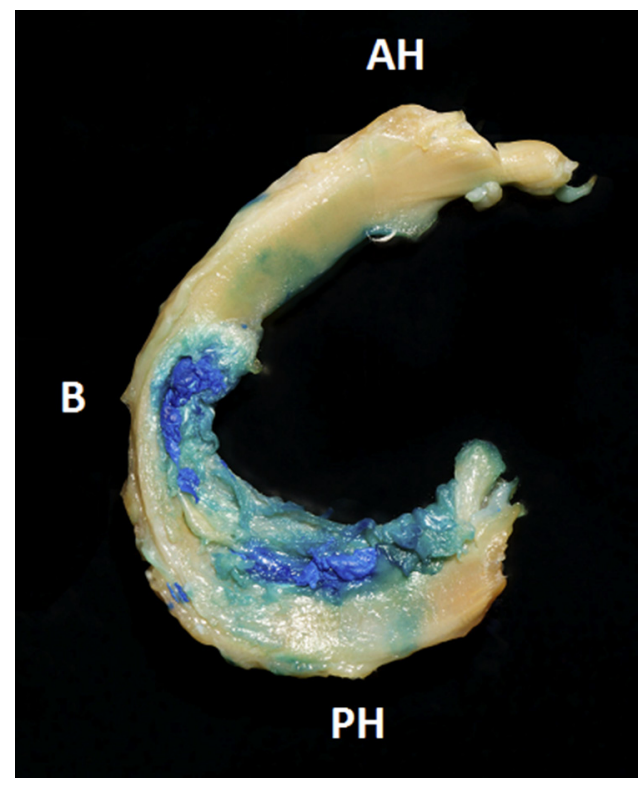

Figure 7. Inferior view of the medial meniscus showing accurate latex deposition into both the body $(\mathrm{B})$ and posterior horn $(\mathrm{PH})$ regions. Similar to the specimen in Figure 4, this specimen exhibited degenerative changes in the body and posterior horns. The distribution of latex within the middle and inner thirds of the meniscus reflect accurate needle placement into these regions as described in the Methods. Note the extension of latex outside of the confines of the meniscus, which was observed in several specimens (See text for discussion). Left $=$ superficial/medial; Right $=$ deep/lateral; Top $=$ anterior; Bottom $=$ posterior; $\mathrm{AH}=$ anterior horn.

the transducer is then translated medially or laterally to the side of interest. The posterior horn of the meniscus is the first soft-tissue, intra-articular structure seen in the tibiofemoral joint after translating medially or laterally off of the PCL. Once the posterior horn is identified, the transducer is rotated $90^{\circ}$ into the anatomical axial plane, yielding a LAX view of the posterior horn.

Seventh, all injections in the current investigation were performed by a single experienced operator. Although the techniques described herein have been easily taught to less-experienced operators, interoperator reliability was not investigated formally in this study. Finally, it is unknown whether there is any

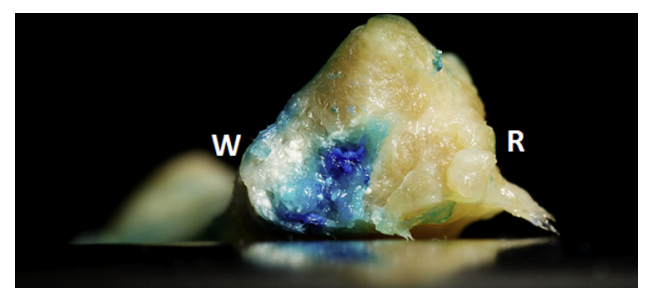

Figure 8. Cross-sectional view of medial meniscus body showing injectate deposition into middle (ie, red-white zone) and inner thirds (ie, white-white zone) of the meniscus. Top = superior; Bottom = inferior; Left = lateral/deep; Right $=$ medial/superficial; $\mathrm{R}=$ red-red zone; $\mathrm{W}=$ white-white zone. clinically relevant iatrogenic injury associated with this technique. Meniscal trephination using an 18-gauge needle is a well-described, long-accepted operative technique to facilitate meniscal healing, and the technique described in this study is similar in principle [19]. Given that primum non nocere remains a guiding principle, this technique should be applied targeting only damaged tissue and using SG so as to minimize the extent of normal tissue traversed.

\section{Conclusions}

SG intrameniscal injections are technically feasible and should be considered when clinically indicated. Further research and clinical experience is necessary to clarify the role of SG intrameniscal injections to reduce pain, improve function, and preserve or regenerate the meniscus in patients with symptomatic acute or chronic degenerative meniscal tears.

\section{Acknowledgments}

We thank the staff of the Mayo Clinic Procedural Skills Laboratory and the noble generosity of the whole-body donors, whose altruistic gift made this study possible.

\section{References}

1. Hunter DJ, Zhang YQ, Niu JB, et al. The association of meniscal pathologic changes with cartilage loss in symptomatic knee osteoarthritis. Arthritis Rheum 2006;54:795-801.

2. Metcalf MH, Barrett GR. Prospective evaluation of 1485 meniscal tear patterns in patients with stable knees. Am J Sports Med 2004; 32:675-680.

3. Frobell R, Cooper R, Morris H, Arendt L. Acute knee injuries. In: Brukner P, Kahn K, eds. Clinical Sports Medicine. Australia: McGraw-Hill; 2009; 635-636.

4. Mautner K, Blazuk J. Where do injectable stem cell treatments apply in treatment of muscle, tendon, and ligament injuries? PM R 2015;7(4 Suppl):S33-S40.

5. Chang KV, Hung CY, Aliwarga F, Wang TG, Han DS, Chen WS. Comparative effectiveness of platelet-rich plasma injections for treating knee joint cartilage degenerative pathology: A systematic review and meta-analysis. Arch Phys Med Rehabil 2014;95:562-575.

6. Vangsness CT Jr, Farr J, Boyd J, Dellaero DT, Mills CR, LeRouxWilliams M. Adult human mesenchymal stem cells delivered via intra-articular injection to the knee following partial medial meniscectomy: A randomized, double-blind, controlled study. J Bone Joint Surg Am 2014;96:90-98.

7. Vangsness CT, McCrum CL. Postmeniscectomy meniscus growth with stem cell: Where are we now? Sports Med Arthrosc 2015;23: 139-142.

8. Taylor SA, Rodeo SA. Augmentation techniques for isolated meniscal tears. Curr Rev Musculoskelet Med 2013;6:95-101.

9. Centeno CJ, Busse D, Kisiday J, Keohan C, Freeman M, Karli D. Regeneration of meniscus cartilage in a knee treated with percutaneously implanted autologous mesenchymal stem cells. Med Hypotheses 2008;71:900-908.

10. Xia XP, Chen HL, Zhou B. Ultrasonography for meniscal injuries in knee joint: A systematic review and meta-analysis. J Sports Med Phys Fitness 2016;56:1179-1187. 
11. Akatsu Y, Yamaguchi S, Mukoyama S, et al. Accuracy of highresolution ultrasound in the detection of meniscal tears and determination of the visible area of menisci. J Bone Joint Surg Am 2015;97:799-806.

12. Rowland G, Mar D, Mclff T, Nelson J. Evaluation of meniscal extrusion with posterior root disruption and repair using ultrasound. Knee 2016;23:627-630.

13. Roos H, Laurén M, Adalberth T, Roos EM, Jonsson K, Lohmander LS. Knee osteoarthritis after meniscectomy: Prevalence of radiographic changes after twenty-one years, compared with matched controls. Arthritis Rheum 1998;41:687-693.

14. Hede A, Larsen E, Sandberg $H$. The long term outcome of open total and partial meniscectomy related to the quantity and site of the meniscus removed. Int Orthop 1992;16:122-125.
15. Bae JY, Park KS, Seon JK, Kwak DS, Jeon I, Song EK. Biomechanical analysis of the effects of medial meniscectomy on degenerative osteoarthritis. Med Biol Eng Comput 2012;50:53-60.

16. Hede A, Larsen E, Sandberg H. Partial versus total meniscectomy. A prospective, randomized study [in Danish]. Ugeskr Laeger 1994; $156: 48-52$.

17. Makris EA, Hadidi P, Athanasiou KA. The knee meniscus: Structurefunction, pathophysiology, current repair techniques, and prospects for regeneration. Biomaterials 2011;32:7411-7431.

18. Hackel JG, Khan U, Loveland DM, Smith J. Sonographically guided posterior cruciate ligament injections: Technique and validation. PM R 2016;8:249-253.

19. Fox JM, Rintz KG, Ferkel RD. Trephination of incomplete meniscal tears. Arthroscopy 1993;9:451-455.

\section{Disclosure}

M.R.B. The Ohio State University Sports Medicine Center \& Department of Physical Medicine and Rehabilitation, Columbus, $\mathrm{OH}$

Disclosure: nothing to disclose

J.L.S. Department of Physical Medicine and Rehabilitation, Mayo Clinic, Rochester, MN

Disclosure: nothing to disclose

D.L. Department of Physical Medicine and Rehabilitation, University of Michigan, Ann Arbor, MI

Disclosure: nothing to disclose
J.S. Department of Physical Medicine and Rehabilitation, Mayo Clinic, W14 Mayo Building, 200 1st St, SW, Rochester, MN 55905. Address correspondence to: J.S.; e-mail: smith.jay@mayo.edu

Disclosures outside this publication: license agreement, stock, and sales royalties, Tenex Health Inc; license agreement, stock, and Chief Medical Officer, Sonex Health, LLC; other, Section Editor, Journal of Ultrasound in Medicine

Funded by Mayo Clinic Institutional Funds.

Submitted for publication August 23, 2016; accepted December 23, 2016. 